\title{
5-Jahresdaten zum Enzymersatz bei M. Fabry
}

\begin{abstract}
Morbus Fabry ist eine sehr seltene genetisch bedingte lysosomale Speicherkrankheit mit hoher klinischer Variabilität, die mit anfallsartigen Parästhesien einhergeht und unter anderem zur Hypertrophie des Herzen und Niereninsuffizienz führt. An der Haut gibt sich die Multisystemerkrankung oft durch multiple Angiokeratome in zentralen Körperregionen zu erkennen. Seit einigen Jahren kann das fehlende bzw. fehlerhafte Enzym per Infusion zugeführt werden.
\end{abstract}

n zwei (jeweils durch den Hersteller finanzierten) internationalen Registern wird der Verlauf der Krankheit ohne und mit Enzymersatztherapie dokumentiert: Im „Fabry Registry“ werden die Daten mit Agalsidase beta behandelter Patienten erfasst; der „Fabry Outcome Survey“ (FOS) hält die Entwicklung von Patienten unter Agalsidase alfa im Vergleich mit dem Verlauf ohne Enzymsubstitution fest.

Eine aktuelle Analyse der FOS-Daten fasst nun Effekte einer fünfährigen Supplementierung des fehlenden Enzyms mit Agalsidase alfa zusammen. Daten von
181 Erwachsenen standen für diesen Zeitraum zur Verfügung.

Bei Patienten, die zu Beginn eine kardiale Hypertrophie aufwiesen, ließ sich eine anhaltende Reduktion der linksventrikulären Masse sowie eine signifikante Verbesserung der Pumpleistung des linken Ventrikels innerhalb von drei Jahren zeigen. Bei Patienten ohne initiale Kardiomyopathie blieben die Messparameter stabil. Auch in Bezug auf den Funktionsverlust der Nieren beobachtete man eine günstige Entwicklung: Je nach Stadium betrugen die Raten von
Stabilisation bzw. Verbesserung zwischen 65 und $75 \%$.

Eine klinisch relevante Linderung der Schmerzintensität erlebten nach fünf Jahren ca. 60\% der befragten Patienten. Außerdem konnte ein signifikanter Gewinn an Lebensqualität festgestellt werden.

$\mathrm{Zu}$ den häufigsten unerwünschten $\mathrm{Ne}$ beneffekten gehörten infusionsbedingte Reaktionen, zerebrovaskuläre Ereignisse, Fieber, Schwindelattacken, transitorische ischämische Attacken, Kopfschmerzen und Übelkeit.

Fazit: Im Vergleich mit nicht substituierten historischen Kontrollgruppen bringt die Langzeittherapie mit Agalsidase alfa den Autoren zufolge substanzielle und anhaltende klinische Verbesserungen.

wpa

Mehta A et al. Enzyme replacement therapy with agalsidase alfa in patients with Fabry's disease: an analysis of registry data. Lancet 374; 2009: 198696.

\section{Stationäre Patienten früh auf Staphylococcus aureus screenen - und sanieren!}

\section{Das Risiko, sich während eines Klinikaufenthalts eine Staphylococcus- aureus-Infektion zuzuziehen, ist für Menschen mit starker nasaler Besiedelung mit diesem Keim drei bis sechs mal so hoch wie für S.-aureus- negative Personen. Über $80 \%$ aller nosokomialen Infektionen durch diesen Erreger sind endogenen Ursprungs.}

\footnotetext{
$M$ it intranasaler Applikation von Mupirocin gelingt zwar nachweislich die Eradikation des Keims, die Datenlage zum Nutzen einer routinemäßigen Mupirocin-Prophylaxe war jedoch bislang nicht eindeutig. In dieser doppelblind kontrollierten niederländischen Studie wurde untersucht, ob die rasche Identifikation von Keimträgern und anschließende Dekolonisation zu einer Eindämmung von Krankenhaus-Infektionen beiträgt.

Von über 6.700 gescreenten Neuzugängen der teilnehmenden Zentren erwiesen sich 1.250 als S.-aureus-positiv. 917 dieser Patienten wurden in die Studie aufgenommen, 808 von ihnen wurden operiert. Sämtliche isolierten Stäm-
}

me waren gegenüber Methicillin und Mupirocin empfindlich.

Unmittelbar nach dem Keimnachweis wurden die Patienten nach dem Zufallsprinzip der Verum- oder Plazebogruppe zugewiesen. Die aktiv Behandelten wandten zweimal täglich das Lokalantibiotikum in der Nase an und duschten einmal täglich mit Chlorhexidin-Seife. Die Studientherapie dauerte fünf Tage. Alle Patienten wurden bis sechs Wochen nach Klinikentlassung beobachtet.

In dieser Zeit zogen sich 3,4\% der Patienten des Verum-Arms eine S.-aureusInfektion zu - gegenüber 7,7\% derjenigen aus der Vergleichsgruppe. Für die Patienten, die sich Dekolonisationsmaßnahmen unterzogen hatten, ergab sich ein relatives Infektionsrisiko von 0,42. Die Zeit bis zum Auftreten einer Infektion war in der Placebogruppe signifikant kürzer als bei den dekolonisierten Patienten. Auch die Dauer des Krankenhaus-Aufenthalts konnte durch die Maßnahmen um fast zwei Tage verkürzt werden.

Im Unterschied zu einigen früheren prospektiven Studien bestimmten die Niederländer den Carrier-Status sofort bei Aufnahme ins Krankenhaus mittels (quantitativer) Echtzeit-PCR. Dieser rasche Nachweis erlaubt eine gezielte Dekolonisation binnen 24 Stunden nach stationärer Aufnahme, also theoretisch noch vor Einwirkung weiterer Risikofaktoren.

Fazit: Die Studie liefert einen soliden Beleg für den präventiven Effekt nasaler und extranasaler Dekolonisation und zeigt auch die ungefähre Größe dieses Effekts: Das Risiko krankenhausassoziierter Infektionen mit S. aureus konnte um nahezu 60\% verringert werden.wpa

Bode LG et al. Preventing surgical-site infections in nasal carriers of Staphylococcus aureus. N Engl J Med 362; 2010: 9-17. 